\title{
On Chebyshev Polynomials, Fibonacci Polynomials, and Their Derivatives
}

\author{
Yang Li \\ Department of Mathematics, Northwest University, Xian, Shaanxi 710127, China \\ Correspondence should be addressed to Yang Li; ly13119143216@126.com
}

Received 6 June 2013; Revised 19 November 2013; Accepted 7 February 2014; Published 13 March 2014

Academic Editor: Vijay Gupta

Copyright (C) 2014 Yang Li. This is an open access article distributed under the Creative Commons Attribution License, which permits unrestricted use, distribution, and reproduction in any medium, provided the original work is properly cited.

We study the relationship of the Chebyshev polynomials, Fibonacci polynomials, and their $r$ th derivatives. We get the formulas for the $r$ th derivatives of Chebyshev polynomials being represented by Chebyshev polynomials and Fibonacci polynomials. At last, we get several identities about the Fibonacci numbers and Lucas numbers.

\section{Introduction}

As we know, the Chebyshev polynomials and Fibonacci polynomials are usually defined as follows: the first kind of Chebyshev polynomials is $T_{n+2}(x)=2 x T_{n+1}(x)-T_{n}(x)$ and $n \geq 0$, with the initial values $T_{0}(x)=1$ and $T_{1}(x)=x$; the second kind of Chebyshev polynomials is $U_{n+2}(x)=$ $2 x U_{n+1}(x)-U_{n}(x)$ and $n \geq 0$, with the initial values $U_{0}(x)=1$ and $U_{1}(x)=2 x$; the Fibonacci polynomials are $F_{n+2}(x)=$ $x F_{n+1}(x)+F_{n}(x)$ and $n \geq 0$ with the initial values $F_{0}(x)=0$ and $F_{1}(x)=1$. From the second-order linear recurrence sequences, we have

$$
\begin{aligned}
T_{n}(x)= & \frac{1}{2}\left[\left(x+\sqrt{x^{2}-1}\right)^{n}+\left(x-\sqrt{x^{2}-1}\right)^{n}\right], \\
U_{n}(x)= & \frac{1}{2 \sqrt{x^{2}-1}} \\
& \times\left[\left(x+\sqrt{x^{2}-1}\right)^{n+1}-\left(x-\sqrt{x^{2}-1}\right)^{n+1}\right], \\
F_{n}(x)= & \frac{1}{2^{n} \sqrt{x^{2}+4}} \\
& \times\left[\left(x+\sqrt{x^{2}+4}\right)^{n}-\left(x-\sqrt{x^{2}+4}\right)^{n}\right] .
\end{aligned}
$$

These polynomials play a very important role in the study of the theory and application of mathematics and they are closely related to the famous Fibonacci numbers $\left\{F_{n}\right\}$ and Lucas numbers $\left\{L_{n}\right\}$ which are defined by the second-order linear recurrence sequences

$$
\begin{gathered}
F_{n+2}=F_{n+1}+F_{n}, \\
L_{n+2}=L_{n+1}+L_{n},
\end{gathered}
$$

where $n \geq 0, F_{0}=0, F_{1}=1, L_{0}=2$, and $L_{1}=1$. Therefore, many authors have investigated these polynomials and got many properties and corollaries. For example, Wu and Zhang [1] have obtained the general formulas involving $F_{n}(x)$

$$
\begin{aligned}
& \left\lfloor\left(\sum_{k=n}^{\infty} \frac{1}{F_{k}(x)}\right)^{-1}\right\rfloor \\
& = \begin{cases}F_{n}(x)-F_{n-1}(x), & \text { if } n \text { is even, } n \geq 2, \\
F_{n}(x)-F_{n-1}(x)-1, & \text { if } n \text { is odd, } n \geq 1,\end{cases} \\
& \left\lfloor\left(\sum_{k=n}^{\infty} \frac{1}{F_{k}^{2}(x)}\right)^{-1}\right\rfloor \\
& = \begin{cases}x F_{n}(x) F_{n-1}(x)-1, & \text { if } n \text { is even, } n \geq 2, \\
x F_{n}(x) F_{n-1}(x), & \text { if } n \text { is odd, } n \geq 1,\end{cases}
\end{aligned}
$$

where $x$ is any positive integer. Wu and Yang [2] studied Chebyshev polynomials and got a lot of properties. 
Recently, several authors also studied the derivatives of these polynomials. For example, Zhang [3] used the $r$ th derivatives of Chebyshev polynomials to solve some calculating problems of the general summations. Falcón and Plaza [4-6] presented many formulas and relations between Fibonacci polynomials and their derivatives. This fact allows them to present a family of integer sequences in a new and direct way.

In this paper, we combine Sergio Falcón and Wenpeng Zhang's ideas. Then we obtain the following theorems and corollaries. These results strengthen the connections of two kinds of polynomials. They are also helpful in dealing with some calculating problems of the general summations or studying some integer sequences.

Theorem 1. For any positive integers, $n$ and $r$, one has the following formulas:

$$
\begin{aligned}
& T_{2 n}^{(2 r-1)}(x) \\
& =\sum_{s=1}^{n-r+1} \sum_{k=r}^{n} \frac{(-1)^{n-k} 2^{2 r} n s(n+k-1) !}{(n-k) !(k-r-s+1) !(k+s+1-r) !} \\
& \quad \times U_{2 s-1}(x), \\
& T_{2 n+1}^{(2 r)}(x) \\
& =\sum_{s=1}^{n-r+1} \sum_{k=r}^{n} \frac{(-1)^{n-k} 2^{2 r}(2 n+1) s(n+k) !}{(n-k) !(k+1+s-r) !(k+1-s-r) !} \\
& \quad \times U_{2 s-1}(x), \\
& T_{2 n+1}^{(2 r-1)}(x) \quad \sum_{s=0}^{n-r+1} \sum_{k=r}^{n} \frac{(-1)^{n-k} 2^{2 r-2}(2 n+1)(2 s+1)(n+k) !}{(n-k) !(k+s+2-r) !(k-s-r+1) !} \\
& \quad \times U_{2 s}(x), \\
& T_{2 n}^{(2 r)}(x) \quad \\
& =\sum_{s=0}^{n-r} \sum_{k=0}^{n} \frac{(-1)^{n-k} \cdot 2^{2 r} \cdot(n+k-1) ! \cdot(2 s+1) \cdot n}{(n-k) !(k-s-r) !(k+s-r+1) !} \\
& \times U_{2 s}(x),
\end{aligned}
$$

where $T_{n}^{(r)}(x)$ denotes the $r$ th derivative of $T_{n}(x)$ with respect to $x$.

Theorem 2. For any positive integers, $n$ and $r$, one has the following formulas:

$$
\begin{aligned}
& T_{2 n+1}^{(2 r-1)}(x) \\
& =\sum_{s=1}^{n-r+1} \sum_{k=r}^{n} \frac{(-1)^{n-k} 2^{2 r-1}(2 n+1)(n+k) ! T_{2 s}(x)}{(n-k) !(s+k+1-r) !(k+1-s-r) !} \\
& \quad+\sum_{k=r}^{n} \frac{(-1)^{n-k} \cdot(2 n+1) \cdot(n+k) !}{2^{2-2 r}(n-k) !(k+1-r) !(k+1-r) !}
\end{aligned}
$$

$$
\begin{aligned}
& T_{2 n}^{(2 r)}(x) \\
& =\sum_{s=1}^{n-r} \sum_{k=r}^{n} \frac{(-1)^{n-k} \cdot 2^{2 r+1} \cdot n(n+k-1) !}{(n-k) !(k-r+s) !(k-r-s) !} T_{2 s}(x) \\
& \quad+\sum_{k=r}^{n} \frac{(-1)^{n-k} \cdot 2^{2 r} \cdot n(n+k-1) !}{(n-k) !(k-r) !(k-r) !}, \\
& T_{2 n+1}^{(2 r)}(x) \\
& =\sum_{s=1}^{n-r+1} \sum_{k=r}^{n} \frac{(-1)^{n-k} 2^{2 r}(n+k) !(2 n+1) T_{2 s-1}(x)}{(n-k) !(s+k-r) !(k-r-s+1) !} \\
& T_{2 n}^{(2 r-1)}(x) \\
& =\sum_{s=1}^{n-r+1} \sum_{k=r}^{n} \frac{(-1)^{n-k} 2^{2 r} n(n+k-1) ! T_{2 s-1}(x)}{(n-k) !(k+s) !(k-r-s+1) !} .
\end{aligned}
$$

Theorem 3. For any positive integers $n$ and $r$ one has the following formulas:

$$
\begin{aligned}
& T_{2 n+1}^{(2 r-1)}(x) \\
&=\sum_{s=1}^{n-r+2} \sum_{k=r}^{n} \frac{(-1)^{n-r-s} 2^{2 k+r-1}(2 s-1)(2 n+1)(n+k) !}{(n-k) !(s+k-r+1) !(k-r-s+2) !} \\
& \quad \times F_{2 s-1}(x), \\
& T_{2 n}^{(2 r)}(x) \quad \sum_{s=1} \sum_{k=r} \frac{(-1)^{n-r-s+1} \cdot 2^{2 k+r}(2 s n-n)(n+k-1) !}{(k+s-r) !(k-r-s+1) !(n-k) !} \\
& T_{2 n}^{(2 r-1)}(x) \\
&=\sum_{s=1}^{n-r+1} \sum_{k=0}^{n} \frac{(-1)^{n-r-s+1} 2^{2 k+r}(n+k-1) ! s n}{(n-k) !(k-r-s+1) !(k+s-r+1) !} \\
& T_{2 n+1}^{(2 r)}(x) \\
&=\sum_{s=1}^{n-r+1} \sum_{k=r}^{n} \frac{(-1)^{n-r-s+1} 2^{2 k+r+1}(2 s n+s)(n+k) !}{(n-k) !(s+k-r+1) !(k-r-s+1) !} \\
& \times F_{2 s}(x), \\
& \times F_{2 s}(x) .
\end{aligned}
$$


Corollary 4. For any positive integers $n, m$, and $r$, one has the following identities:

$$
\begin{aligned}
& T_{2 n}^{(2 r-1)}\left(\frac{i^{m} L_{m}}{2}\right) \\
& =\sum_{s=1}^{n-r+1} \sum_{k=r}^{n} \frac{(-1)^{s m+n-k+m} \cdot i^{m} \cdot 2^{2 r} n s(n+k-1) ! F_{2 s m}}{(n-k) !(k-r-s+1) !(k+s+1-r) ! F_{m}}, \\
& T_{2 n+1}^{(2 r)}\left(\frac{i^{m} L_{m}}{2}\right) \\
& =\sum_{s=1}^{n-r+1} \sum_{k=r}^{n} \frac{(-1)^{s m+n+m-k} 2^{2 r}(2 n+1) i^{m} s(n+k) ! F_{2 m s}}{(n-k) !(k+1+s-r) !(k+1-s-r) ! F_{m}} \\
& T_{2 n+1}^{(2 r-1)}\left(\frac{i^{m} L_{m}}{2}\right) \\
& =\sum_{s=0}^{n-r+1} \sum_{k=r}^{n} \frac{(-1)^{s m+n} 2^{2 r-2}(2 n+1)(2 s+1)(n+k) ! F_{m(2 s+1)}}{(n-k) !(k+s+2-r) !(k-s-r+1) ! F_{m}}, \\
& T_{2 n}^{(2 r)}\left(\frac{i^{m} L_{m}}{2}\right) \\
& \quad=\sum_{s=0}^{n-r} \sum_{k=0}^{n} \frac{(-1)^{s m+n-k} \cdot 2^{2 r}(n+k-1) !(2 s+1) n F_{m(2 s+1)}}{(n-k) !(k-s-r) !(k+s-r+1) ! F_{m}},
\end{aligned}
$$

where $i$ denotes the square root of -1 .

Corollary 5. For any positive integers $n, m$, and $r$, one has the following identities:

$$
\begin{aligned}
& T_{2 n+1}^{(2 r-1)}\left(\frac{i^{m} L_{m}}{2}\right) \\
& =\sum_{s=1}^{n-r+1} \sum_{k=r}^{n} \frac{(-1)^{s m+n-k} 2^{2 r-2}(2 n+1)(n+k) ! L_{2 s m}}{(n-k) !(s+k+1-r) !(k+1-s-r) !} \\
& \quad+\sum_{k=r}^{n} \frac{(-1)^{n-k} \cdot(2 n+1) \cdot(n+k) !}{2^{2-2 r}(n-k) !(k+1-r) !(k+1-r) !} \\
& T_{2 n}^{(2 r)}\left(\frac{i^{m} L_{m}}{2}\right) \\
& =\sum_{s=1}^{n-r} \sum_{k=r}^{n} \frac{(-1)^{s m+n-k} \cdot 2^{2 r} \cdot n(n+k-1) !}{(n-k) !(k-r+s) !(k-r-s) !} L_{2 s m} \\
& \quad+\sum_{k=r}^{n} \frac{(-1)^{n-k} \cdot 2^{2 r} \cdot n(n+k-1) !}{(n-k) !(k-r) !(k-r) !}
\end{aligned}
$$

$$
\begin{aligned}
& T_{2 n+1}^{(2 r)}\left(\frac{i^{m} L_{m}}{2}\right) \\
& =\sum_{s=1}^{n-r+1} \sum_{k=r}^{n} \frac{(-1)^{s m+n-k} 2^{2 r-1}(n+k) !(2 n+1) L_{2 s m-m}}{i^{m}(n-k) !(s+k-r) !(k-r-s+1) !} \\
& T_{2 n}^{(2 r-1)}\left(\frac{i^{m} L_{m}}{2}\right) \\
& =\sum_{s=1}^{n-r+1} \sum_{k=r}^{n} \frac{(-1)^{s m+n-k} 2^{2 r-1} n(n+k-1) ! L_{2 s m-m}}{i^{m}(n-k) !(k+s-r) !(k-r-s+1) !}
\end{aligned}
$$

\section{Some Lemmas}

Lemma 6. For any nonnegative integers $m$ and $n$, one has the following identities:

$$
\begin{gathered}
\int_{-1}^{1} \frac{T_{m}(x) T_{n}(x)}{\sqrt{1-x^{2}}} d x= \begin{cases}0, & m \neq n, \\
\frac{\pi}{2}, & m=n>0, \\
\pi, & m=n=0,\end{cases} \\
\int_{-1}^{1} U_{m}(x) U_{n}(x) \sqrt{1-x^{2}} d x= \begin{cases}0, & m \neq n, \\
\frac{\pi}{2}, & m=n,\end{cases} \\
T_{n}(\cos \theta)=\cos n \theta, \\
U_{n}(\cos \theta)=\frac{\sin (n+1) \theta}{\sin \theta} .
\end{gathered}
$$

Proof. See [7].

Lemma 7. For any positive integers $m$ and $n$, one has the following identities:

$$
\begin{aligned}
& U_{n}\left(\frac{i}{2}\right)=i^{n} F_{n+1}, \\
& T_{n}\left(\frac{i}{2}\right)=\frac{i^{n}}{2} L_{n+1} .
\end{aligned}
$$

Proof. See [3].

Lemma 8. For any positive integer $n$, one has

$$
\begin{gathered}
T_{n}\left(T_{m}(x)\right)=T_{n m}(x), \\
U_{n}\left(T_{m}(x)\right)=\frac{U_{m(n+1)-1}(x)}{U_{m-1}(x)} .
\end{gathered}
$$

Proof. See [3]. 
Lemma 9. For any positive integers $n$ and $r$, one has

$$
\begin{aligned}
& T_{2 n}^{(r)}(x)=\sum_{k=\lceil r / 2\rceil}^{n} \frac{(-1)^{n-k} 2^{2 k} n(n+k-1) !}{(n-k) !(2 k-r) !} x^{2 k-r}, \\
& T_{2 n+1}^{(r)}(x) \\
& =\sum_{k=\lceil r / 2\rceil}^{n} \frac{(-1)^{n-k} 2^{2 k}(2 n+1)(n+k) !}{(n-k) !(2 k+1-r) !} x^{2 k+1-r} .
\end{aligned}
$$

Proof. From Theorem 2 of [2], we can get the following result easily:

$$
\begin{aligned}
T_{2 n}(x)=\sum_{k=0}^{n}( & -1)^{n-k} \\
& \times\left[2^{2 k}\left(\begin{array}{c}
n+k \\
n-k
\end{array}\right)-2^{2 k-1}\left(\begin{array}{c}
n+k-1 \\
2 k-1
\end{array}\right)\right] x^{2 k} \\
= & \sum_{k=0}^{n}(-1)^{n-k} \cdot 2^{2 k-1} \\
& \times\left[\frac{2(n+k) !}{(2 k) !(n-k) !}-\frac{(n+k-1) !}{(2 k-1) !(n-k) !}\right] x^{2 k} \\
= & \sum_{k=0}^{n} \frac{n(-1)^{n-k} 2^{2 k}(n+k-1) !}{(2 k) !(n-k) !} x^{2 k} .
\end{aligned}
$$

From Theorem 2 of [2], we know

$$
\begin{aligned}
T_{2 n+1}(x)=\sum_{k=0}^{n}(-1)^{n-k} & \\
& \times\left[2^{2 k+1}\left(\begin{array}{c}
n+k+1 \\
n-k
\end{array}\right)-2^{2 k}\left(\begin{array}{c}
n+k \\
2 k
\end{array}\right)\right] x^{2 k+1} .
\end{aligned}
$$

In the similar way, we can get the following result easily:

$$
T_{2 n+1}(x)=\sum_{k=0}^{n} \frac{(-1)^{n-k}(2 n+1) 2^{2 k}(n+k) !}{(2 k+1) !(n-k) !} x^{2 k+1} .
$$

If we derive both sides of the above properties $r$ th times, we will get

$$
\begin{aligned}
& T_{2 n}^{(r)}(x) \\
& \quad=\sum_{k=\lceil r / 2\rceil}^{n} \frac{(-1)^{n-k} 2^{2 k} n(n+k-1) !}{(n-k) !(2 k-r) !} x^{2 k-r}, \\
& T_{2 n+1}^{(r)}(x) \\
& \quad=\sum_{k=\lceil r / 2\rceil}^{n} \frac{(-1)^{n-k} 2^{2 k}(2 n+1)(n+k) !}{(n-k) !(2 k+1-r) !} x^{2 k+1-r} .
\end{aligned}
$$

This proves Lemma 9.
Lemma 10. For any positive integers $n$ and $r$, let

$$
\begin{gathered}
T_{2 n}^{(r)}(x)=\sum_{s=0}^{+\infty} a_{2 n, r, s} U_{s}(x), \\
T_{2 n+1}^{(r)}(x)=\sum_{s=0}^{+\infty} a_{2 n+1, r, s} U_{s}(x),
\end{gathered}
$$

where $T_{n}^{(r)}(x)$ denotes the rth derivative of $T_{n}(x)$ with respect to $x$. Then one can get

$$
a_{2 n, r, s}=\left\{\begin{array}{l}
\sum_{k=\lceil r / 2\rceil}^{n} \frac{(-1)^{n-k} 2^{2 k+1} n(n+k-1) !(s+1)}{(n-k) !(2 k-s-r) ! !(2 k+s-r+2) ! !}, \\
0, \quad \text { otherwise, }
\end{array}\right.
$$

$$
\begin{aligned}
& a_{2 n+1, r, s}
\end{aligned}
$$

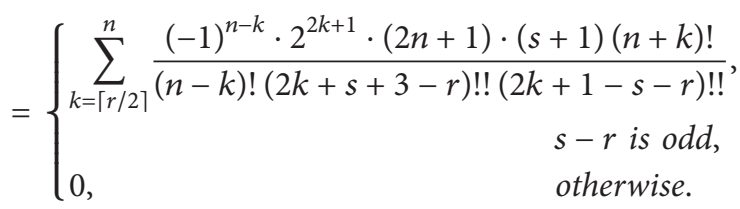

Proof. To begin with, we multiply $\sqrt{1-x^{2}} U_{m}(x)$ to both sides of the following identity:

$$
T_{n}^{(r)}(x)=\sum_{s=0}^{+\infty} a_{n r s} U_{s}(x),
$$

and then integrate it from -1 to 1 . Applying property (10), we can get

$$
\begin{aligned}
& \int_{-1}^{1} \sqrt{1-x^{2}} T_{n}^{(r)}(x) U_{m}(x) d x \\
& =\sum_{s=0}^{\infty} \int_{-1}^{1} a_{n, r, s} \sqrt{1-x^{2}} U_{m}(x) U_{s}(x) d x \\
& =\frac{\pi}{2} a_{n, r, m}
\end{aligned}
$$

and then we have

$$
a_{n r m}=\frac{2}{\pi} \int_{-1}^{1} \sqrt{1-x^{2}} T_{n}^{(r)}(x) U_{m}(x) d x .
$$

We define

$$
w_{n k}=\frac{2}{\pi} \int_{0}^{\pi} \cos ^{n} \theta \sin (k+1) \theta \sin \theta d \theta .
$$

From [8], we know

$$
w_{n k}= \begin{cases}\frac{2(k+1) n !}{(n+k+2) ! !(n-k) ! !}, & k+n \text { is even, } n \geq k \\ 0, & \text { otherwise }\end{cases}
$$


where $n$ and $k$ are any nonnegative integers. Let $x=\cos \theta$; then we can get the following identity by applying property (10):

$$
\begin{aligned}
a_{n r m} & =\frac{2}{\pi} \int_{0}^{\pi} T_{n}^{(r)}(\cos \theta) U_{m}(\cos \theta) \sin ^{2} \theta d \theta \\
& =\frac{2}{\pi} \int_{0}^{\pi} T_{n}^{(r)}(\cos \theta) \sin (m+1) \theta \sin \theta d \theta
\end{aligned}
$$

According to Lemma 9 and property (27), we have

$$
\begin{aligned}
a_{2 n, r, m}= & \frac{2}{\pi} \int_{0}^{\pi} T_{2 n}^{(r)}(\cos \theta) \sin (m+1) \theta \sin \theta d \theta \\
= & \frac{2}{\pi} \sum_{k=\lceil r / 2\rceil}^{n} \frac{2^{2 k} \cdot n \cdot(n+k-1) !}{(-1)^{n-k}(n-k) !(2 k-r) !} \\
& \times \int_{0}^{\pi} \cos ^{2 k-r} \theta \sin (m+1) \theta \sin \theta d \theta \\
= & \sum_{k=[r / 2\rceil}^{n} \frac{2^{2 k} \cdot n \cdot(n+k-1) !}{(-1)^{n-k}(n-k) !(2 k-r) !} w_{2 k-r, m} .
\end{aligned}
$$

Then we have $a_{2 n, r, m}=0$ if $m-r$ is odd. If $m-r$ is even, we have

$$
\begin{aligned}
a_{2 n, r, m}= & \sum_{k=\lceil r / 2\rceil}^{n} \frac{2^{2 k} \cdot n \cdot(n+k-1) !}{(-1)^{n-k}(n-k) !(2 k-r) !} \\
& \cdot \frac{2(m+1)(2 k-r) !}{(2 k-m-r) ! !(2 k+m+2-r) ! !} \\
= & \sum_{k=\lceil r / 2\rceil}^{n} \frac{(-1)^{n-k} 2^{2 k+1} n(n+k-1) !(m+1)}{(n-k) !(2 k-m-r) ! !(2 k+m-r+2) ! !} .
\end{aligned}
$$

This proves property (21). In the similar way, we have $a_{2 n+1, r, m}=0$ if $m-r$ is even. If $m-r$ is odd, we have

$$
\begin{aligned}
& a_{2 n+1, r, m} \\
& \quad=\sum_{k=[r / 2]}^{n} \frac{(-1)^{n-k} 2^{2 k}(2 n+1)(n+k) !}{(n-k) !(2 k+1-r) !} w_{2 k+1-r, m} \\
& \quad=\sum_{k=[r / 2]}^{n} \frac{(-1)^{n-k} \cdot 2^{2 k+1} \cdot(2 n+1) \cdot(m+1)(n+k) !}{(n-k) !(2 k+m+3-r) ! !(2 k+1-m-r) ! !} .
\end{aligned}
$$

That is property (22). This proves Lemma 10.

Lemma 11. For any positive integers $n$ and $r$, let

$$
\begin{gathered}
T_{2 n}^{(r)}(x)=\frac{1}{2} b_{2 n, r, 0} T_{0}(x)+\sum_{s=1}^{+\infty} b_{2 n, r, s} T_{s}(x), \\
T_{2 n+1}^{(r)}(x)=\frac{1}{2} b_{2 n+1, r, 0} T_{0}(x)+\sum_{s=1}^{+\infty} b_{2 n+1, r, s} T_{s}(x) .
\end{gathered}
$$

Then one can get

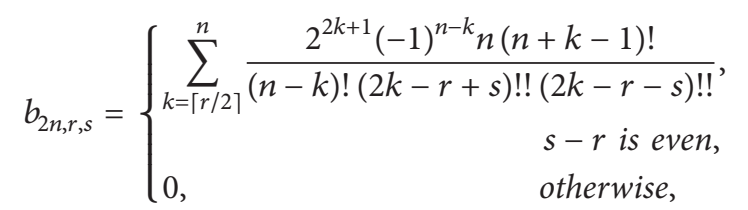

$$
\begin{aligned}
& b_{2 n+1, r, s} \\
& = \begin{cases}\sum_{k=\lceil r / 2\rceil}^{n} \frac{(-1)^{n-k} \cdot 2^{2 k+1} \cdot(2 n+1)(n+k) !}{(n-k) !(s+2 k+1-r) ! !(2 k+1-r-s) ! !}, \\
0, & \begin{array}{l}
\text { s }-r \text { is odd }, \\
\text { otherwise. }
\end{array}\end{cases}
\end{aligned}
$$

Proof. In order to prove property (22) we must multiply $T_{m}(x) / \sqrt{1-x^{2}}$ to both sides of the following identity:

$$
T_{n}^{(r)}(x)=\frac{1}{2} b_{n, r, 0} T_{0}(x)+\sum_{s=1}^{+\infty} b_{n, r, s} T_{s}(x),
$$

and then integrate it from -1 to 1 . Applying property (9) we can get

$$
\begin{aligned}
\int_{-1}^{1} \frac{T_{n}^{(r)}(x) T_{m}(x)}{\sqrt{1-x^{2}}} d x= & \int_{-1}^{1} \frac{b_{n r 0} T_{0}(x) T_{m}(x)}{2 \sqrt{1-x^{2}}} d x \\
& +\sum_{k=1}^{\infty} \int_{-1}^{1} \frac{b_{n r k} T_{m}(x) T_{n}(x)}{\sqrt{1-x^{2}}} d x \\
& =\frac{\pi}{2} b_{n r m}
\end{aligned}
$$

and then we have

$$
b_{n r m}=\frac{2}{\pi} \int_{-1}^{1} \frac{T_{n}^{(r)}(x) T_{m}(x)}{\sqrt{1-x^{2}}} d x .
$$

We define

$$
q_{n k}=\frac{2}{\pi} \int_{0}^{\pi} \cos ^{n} \theta \cos k \theta d \theta .
$$

From [8], we know

$$
q_{n k}= \begin{cases}\frac{2 n !}{(n+k) ! !(n-k) ! !}, & k+n \text { is even, } n \geq k, \\ 0, & \text { otherwise, }\end{cases}
$$

where $n$ and $k$ are any nonnegative integers. Let $x=\cos \theta$; then we can get the following identity by applying property (10):

$$
\begin{aligned}
b_{n r m} & =\frac{2}{\pi} \int_{0}^{\pi} \frac{T_{m}(\cos \theta) T_{n}^{(r)}(\cos \theta)}{\sin \theta} \sin \theta d \theta \\
& =\frac{2}{\pi} \int_{0}^{\pi} T_{m}(\cos \theta) T_{n}^{(r)}(\cos \theta) d \theta .
\end{aligned}
$$


According to Lemma 9 and property (39), we have

$$
\begin{aligned}
b_{2 n, r, m} & =\frac{2}{\pi} \int_{0}^{\pi} T_{2 n}^{(r)}(\cos \theta) \cos m \theta d \theta \\
& =\sum_{k=\lceil r / 2\rceil}^{n} \frac{2^{2 k} n(n+k-1) ! q_{2 k-r, m}}{(-1)^{n-k}(n-k) !(2 k-r) !}
\end{aligned}
$$

so we have $b_{2 n, r, m}=0$ if $m-r$ is odd. If $m-r$ is even, we have

$$
\begin{aligned}
b_{2 n, r, m}= & \sum_{k=\lceil r / 2\rceil}^{n} \frac{2^{2 k} n(n+k-1) !}{(n-k) !(2 k-r) !} \\
& \cdot \frac{2(-1)^{n-k}(2 k-r) !}{(2 k-r+m) ! !(2 k-r-m) ! !} \\
= & \sum_{k=\lceil r / 2\rceil}^{n} \frac{2^{2 k+1}(-1)^{n-k} n(n+k-1) !}{(n-k) !(2 k-r+m) ! !(2 k-r-m) ! !} .
\end{aligned}
$$

This proves property (33). In the similar way we have $b_{2 n+1, r, m}=0$ if $m-r$ is even. If $m-r$ is odd, we have

$$
\begin{aligned}
& b_{2 n+1, r, m} \\
& =\sum_{k=\lceil r / 2\rceil}^{n} \frac{2^{2 k}(2 n+1)(n+k) ! q_{2 k+1-r, m}}{(-1)^{n-k}(n-k) !(2 k+1-r) !} \\
& =\sum_{k=\lceil r / 2\rceil}^{n} \frac{(-1)^{n-k} \cdot 2^{2 k+1} \cdot(2 n+1)(n+k) !}{(n-k) !(m+2 k+1-r) ! !(2 k+1-r-m) ! !} .
\end{aligned}
$$

That is property (34). This proves Lemma 11.

Lemma 12. For any positive integers $m$ and $n$, one has the following identities:

$$
\begin{gathered}
F_{n}(2 i \cos \theta)=\frac{i^{n+3} \sin n \theta}{\sin \theta}, \\
\int_{-1}^{1} \sqrt{x^{2}+4} F_{m}(x) F_{n}(x) d x= \begin{cases}2 i^{2 m-1} \pi, & m=n>0, \\
0, & \text { otherwise. }\end{cases}
\end{gathered}
$$

Proof. As we know,

$$
\begin{aligned}
F_{n}(x)= & \frac{1}{2^{n} \sqrt{x^{2}+4}} \\
& \times\left[\left(x+\sqrt{x^{2}+4}\right)^{n}-\left(x-\sqrt{x^{2}+4}\right)^{n}\right] .
\end{aligned}
$$

Let $x=2 i \cos \theta$; then we have

$$
\begin{aligned}
F_{n}(2 i \cos \theta) & =\frac{1}{2 \sin \theta}\left[(i \cos \theta+\sin \theta)^{n}-(i \cos \theta-\sin \theta)^{n}\right] \\
& =\frac{1}{2 \sin \theta}\left(i^{n} e^{-i n \theta}-i^{n} e^{i n \theta}\right) \\
& =\frac{i^{n}}{2 \sin \theta}(\cos n \theta-i \sin n \theta-\cos n \theta-i \sin n \theta) \\
& =\frac{i^{n+3} \sin n \theta}{\sin \theta} .
\end{aligned}
$$

This proves property (44). Let $x=2 i \cos \theta$ in the following identity:

$$
A=\int_{-2 i}^{2 i} \sqrt{x^{2}+4} F_{m}(x) F_{n}(x) d x
$$

then we can get

$$
\begin{aligned}
A & =\int_{0}^{\pi} 2 \sin \theta F_{n}(2 i \cos \theta) F_{m}(2 i \cos \theta) 2 i \sin \theta d \theta \\
& =\int_{0}^{\pi} 4 i \sin ^{2} \theta \frac{i^{n+3} \sin n \theta}{\sin \theta} \frac{i^{m+3} \sin m \theta}{\sin \theta} d \theta \\
& =4 i^{n+m-1} \int_{0}^{\pi} \sin n \theta \sin m \theta d \theta \\
& =2 i^{n+m-1} \int_{0}^{\pi} \cos (n-m) \theta-\cos (m+n) \theta d \theta .
\end{aligned}
$$

Then we can get property (45). This proves Lemma 12 .

Lemma 13. For any positive integer $n$, let

$$
\begin{aligned}
T_{2 n}^{(r)}(x) & =\sum_{s=1}^{+\infty} c_{n, r, s} F_{s}(x), \\
T_{2 n+1}^{(r)}(x) & =\sum_{s=1}^{+\infty} c_{n, r, s} F_{s}(x) ;
\end{aligned}
$$

then we can get

$c_{2 n, r, s}= \begin{cases}\sum_{k=\lceil r / 2\rceil}^{n} \frac{2^{4 k-r+1} n s(n+k-1) ! i^{1-r-s}(-1)^{n}}{(n-k) !(2 k-r-s+1) ! !(2 k-r+s+1) ! !}, \\ s-r \text { is odd }, \\ 0, \quad \text { otherwise, }\end{cases}$

$c_{2 n+1, r, s}$

$$
=\left\{\begin{array}{l}
\sum_{k=\lceil r / 2\rceil}^{n} \frac{(-1)^{n} 2^{4 k+2-r}(2 s n+s) i^{2-r-s}(n+k) !}{(n-k) !(2 k+s-r+2) ! !(2 k-r-s+2) ! !} \\
0, \quad \text { otherwise. }
\end{array}\right.
$$


Proof. At first, we multiply $\sqrt{x^{2}+4} F_{m}(x)$ to both sides of the following identity:

$$
T_{n}^{(r)}(x)=\sum_{s=1}^{+\infty} c_{n, r, s} F_{s}(x)
$$

and then integrate it from $-2 i$ to $2 i$; we can get the following identity by applying Lemma 12, where $m$ is any positive integer. Consider

$$
\begin{aligned}
\int_{-2 i}^{2 i} & \sqrt{x^{2}+4} F_{m}(x) T_{n}^{(r)}(x) d x \\
= & \sum_{s=1}^{\infty} \int_{-2 i}^{2 i} c_{n, r, s} \sqrt{x^{2}+4} F_{s}(x) F_{m}(x) d x \\
= & 2 i^{2 m-1} \pi c_{n, r, m} ;
\end{aligned}
$$

then we have

$$
c_{n, r, m}=\frac{(-i)^{2 m-1}}{2 \pi} \int_{-2 i}^{2 i} \sqrt{x^{2}+4} F_{m}(x) T_{n}^{(r)}(x) d x .
$$

Let $x=\cos \theta$; then we can get the following identity by applying Lemma 12:

$$
\begin{aligned}
c_{n, r, m}= & \frac{(-i)^{2 m-1}}{2 \pi} \\
& \times \int_{0}^{\pi} T_{n}^{(r)}(2 i \cos \theta) F_{m}(2 i \cos \theta) 4 i \sin ^{2} \theta d \theta \\
= & \frac{i^{2 m+2}}{2 \pi} \int_{0}^{\pi} T_{n}^{(r)}(2 i \cos \theta) \cdot \frac{i^{m+3} \sin m \theta}{\sin \theta} \cdot(2 \sin \theta)^{2} d \theta \\
= & \frac{2 i^{3 m+1}}{\pi} \int_{0}^{\pi} T_{n}^{(r)}(2 i \cos \theta) \sin m \theta \sin \theta d \theta .
\end{aligned}
$$

According to property (27), we have

$$
\begin{aligned}
& c_{2 n, r, m}= \sum_{k=\lceil r / 2\rceil}^{n} \frac{2^{2 k+1} \cdot n i^{3 m+1}(n+k-1) !}{(-1)^{n-k}(n-k) !(2 k-r) ! \pi} \\
& \times \int_{0}^{\pi}(2 i \cos \theta)^{2 k-r} \sin m \theta \sin \theta d \theta \\
&= \sum_{k=\lceil r / 2\rceil}^{n} \frac{2^{4 k+1} n(n+k-1) ! i^{1-r-m}}{(-1)^{n}(n-k) !(2 k-r) ! 2^{r} \pi} \\
& \quad \times \int_{0}^{\pi} \cos ^{2 k-r} \theta \sin m \theta \sin \theta d \theta \\
&=\sum_{k=\lceil r / 2\rceil}^{n} \frac{2^{4 k-r} n(n+k-1) ! i^{1-r-m}}{(-1)^{n}(n-k) !(2 k-r) !} w_{2 k-r, m-1}
\end{aligned}
$$

so we have $c_{2 n, r, m}=0$ if $m-r$ is even. If $m-r$ is odd, we can get

$$
\begin{aligned}
& c_{2 n, r, m} \\
& =\sum_{k=\lceil r / 2\rceil}^{n} \frac{2^{4 k-r} n(n+k-1) ! i^{1-r-m}}{(-1)^{n}(n-k) !(2 k-r) !} \\
& \quad \times \frac{2 m(2 k-r) !}{(2 k-r-m+1) ! !(2 k-r+m+1) ! !} \\
& =\sum_{k=\lceil r / 2\rceil}^{n} \frac{2^{4 k-r+1} \cdot n m(n+k-1) ! i^{1-r-m}(-1)^{n}}{(n-k) !(2 k-r-m+1) ! !(2 k-r+m+1) ! !} .
\end{aligned}
$$

In the similar way, we have $c_{2 n+1, r, m}=0$ if $m-r$ is odd. If $m-r$ is even, we can get

$$
\begin{aligned}
& c_{2 n+1, r, m} \\
& \quad=\sum_{k=\lceil r / 2\rceil}^{n} \frac{2^{4 k+1-r}(2 n+1) i^{2-r-m}(n+k) !}{(-1)^{n}(n-k) !(2 k+1-r) !} w_{2 k+1-r, m-1} \\
& =\sum_{k=\lceil r / 2\rceil}^{n} \frac{(-1)^{n} 2^{4 k+2-r}(2 m n+m) i^{2-r-m}(n+k) !}{(n-k) !(2 k+m-r+2) ! !(2 k-r-m+2) ! !} .
\end{aligned}
$$

This proves Lemma 13.

\section{Proof of the Theorems and Corollaries}

In this section, we will prove our theorems and corollaries. First of all, we can prove all the theorems from Lemmas 10, 11, and 13 easily. Then we prove our corollaries.

Proof of Corollary 4. Let $x=T_{m}(x)$ in Theorem 1 . We can get the following properties from Lemma 8:

$$
\begin{aligned}
& T_{2 n}^{(2 r-1)}\left(T_{m}(x)\right) \\
& =\sum_{s=1}^{n-r+1} \sum_{k=r}^{n} \frac{(-1)^{n-k} 2^{2 r} n s(n+k-1) ! U_{2 s m-1}(x)}{(n-k) !(k-r-s+1) !(k+s+1-r) ! U_{m-1}(x)}, \\
& T_{2 n+1}^{(2 r)}\left(T_{m}(x)\right) \\
& =\sum_{s=1}^{n-r+1} \sum_{k=r}^{n} \frac{(-1)^{n-k} 2^{2 r}(2 n+1) s(n+k) ! U_{2 s m-1}(x)}{(n-k) !(k+1+s-r) !(k+1-s-r) ! U_{m-1}(x)}, \\
& T_{2 n+1}^{(2 r-1)}\left(T_{m}(x)\right) \\
& =\sum_{s=0}^{n+1-r} \sum_{k=r}^{n} \frac{(-1)^{n-k} 2^{2 r-2}(2 n+1)(2 s+1)(n+k) ! U_{m(2 s+1)-1}(x)}{(n-k) !(k+s+2-r) !(k-s-r+1) ! U_{m-1}(x)}, \\
& T_{2 n}^{(2 r)}\left(T_{m}(x)\right) \\
& \quad=\sum_{s=0}^{n-r} \sum_{k=0}^{n} \frac{(-1)^{n-k} \cdot 2^{2 r}(n+k-1) !(2 s+1) n U_{m(2 s+1)-1}(x)}{(n-k) !(k-s-r) !(k+s-r+1) ! U_{m-1}(x)} .
\end{aligned}
$$


Then, taking $x=i / 2$ in the above identities, according to Lemma 7, we can get Corollary 4.

Proof of Corollary 5. Let $x=T_{m}(x)$ in Theorem 2. We can get the following properties from Lemma 8:

$$
\begin{aligned}
& T_{2 n+1}^{(2 r-1)}\left(T_{m}(x)\right) \\
& =\sum_{s=1}^{n-r+1} \sum_{k=r}^{n} \frac{(-1)^{n-k} 2^{2 r-1}(2 n+1)(n+k) ! T_{2 s m}(x)}{(n-k) !(s+k+1-r) !(k+1-s-r) !} \\
& \quad+\sum_{k=r}^{n} \frac{(-1)^{n-k} \cdot(2 n+1) \cdot(n+k) !}{2^{2-2 r}(n-k) !(k+1-r) !(k+1-r) !}, \\
& T_{2 n}^{(2 r)}\left(T_{m}(x)\right) \\
& =\sum_{s=1}^{n-r} \sum_{k=r}^{n} \frac{(-1)^{n-k} \cdot 2^{2 r+1} \cdot n(n+k-1) !}{(n-k) !(k-r+s) !(k-r-s) !} T_{2 s m}(x) \\
& \quad+\sum_{k=r}^{n} \frac{(-1)^{n-k} \cdot 2^{2 r} \cdot n(n+k-1) !}{(n-k) !(k-r) !(k-r) !}, \\
& T_{2 n+1}^{(2 r)}\left(T_{m}(x)\right) \\
& =\sum_{2 n}^{(2 r-1)}\left(T_{m}^{n-r+1} \sum_{s=1}^{n} \frac{(-1)^{n-k} 2^{2 r}(n+k) !(2 n+1) T_{2 s m-m}(x)}{(n-k) !(s+k-r) !(k-r-s+1) !},\right. \\
& \sum_{s=1}^{n-r+1} \frac{(-1)^{n-k} 2^{2 r} n(n+k-1) ! T_{2 s m-m}(x)}{(n-k) !(k+s-r) !(k-r-s+1) !} .
\end{aligned}
$$

Then, taking $x=i / 2$ in the above identities, according to Lemma 7, we can get Corollary 5 .

\section{Conflict of Interests}

The author declares that he has no conflict of interests in this paper.

\section{Acknowledgments}

The author would like to thank the referee for his very helpful and detailed comments, which have significantly improved the presentation of this paper. This work is supported by the N.S.F. (11371291, 61202437) and S.R.F.D.P. (20136101110014) of China.

\section{References}

[1] Z. Wu and W. Zhang, "The sums of the reciprocals of Fibonacci polynomials and Lucas polynomials," Journal of Inequalities and Applications, vol. 2012, article 134, 2012.

[2] X. Wu and G. Yang, "The general formula of Chebyshev polynomials," Journal of Wuhan Transportation University, vol. 24, pp. 573-576, 2000.

[3] W. Zhang, "Some identities involving the Fibonacci numbers and Lucas numbers," The Fibonacci Quarterly, vol. 42, no. 2, pp. 149-154, 2004.
[4] S. Falcón and Á. Plaza, "On $k$-Fibonacci sequences and polynomials and their derivatives," Chaos, Solitons \& Fractals, vol. 39, no. 3, pp. 1005-1019, 2009.

[5] S. Falcón and Á. Plaza, "The $k$-Fibonacci sequence and the Pascal 2-triangle," Chaos, Solitons \& Fractals, vol. 33, no. 1, pp. 38-49, 2007.

[6] S. Falcón and Á. Plaza, "The $k$-Fibonacci hyperbolic functions," Chaos, Solitons \& Fractals, vol. 38, no. 2, pp. 409-420, 2008.

[7] U. W. Hochstrasser, "Orthogonal polynomials," in Handbook of Mathematical Functions, with Formulas, Graphs, and Mathematical Tables, M. Abramowitz and I. A. Stegun, Eds., chapter 22, pp. 771-802, Dover, New York, NY, USA, 1965.

[8] R. Ma and W. Zhang, "Several identities involving the Fibonacci numbers and Lucas numbers," The Fibonacci Quarterly, vol. 45, no. 2, pp. 164-170, 2007. 


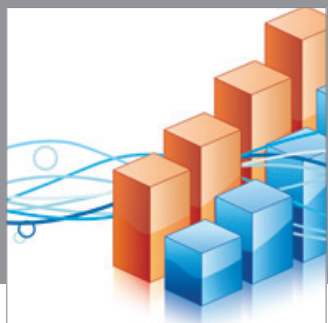

Advances in

Operations Research

mansans

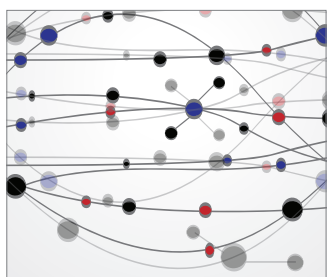

The Scientific World Journal
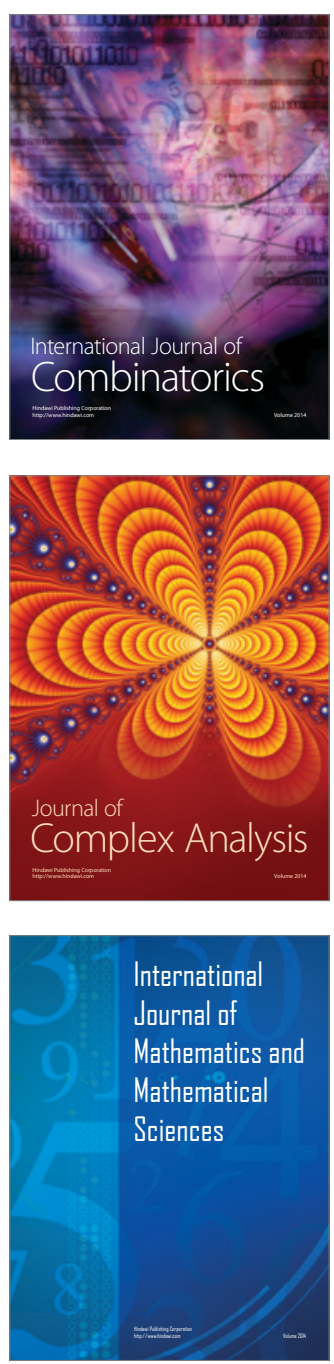
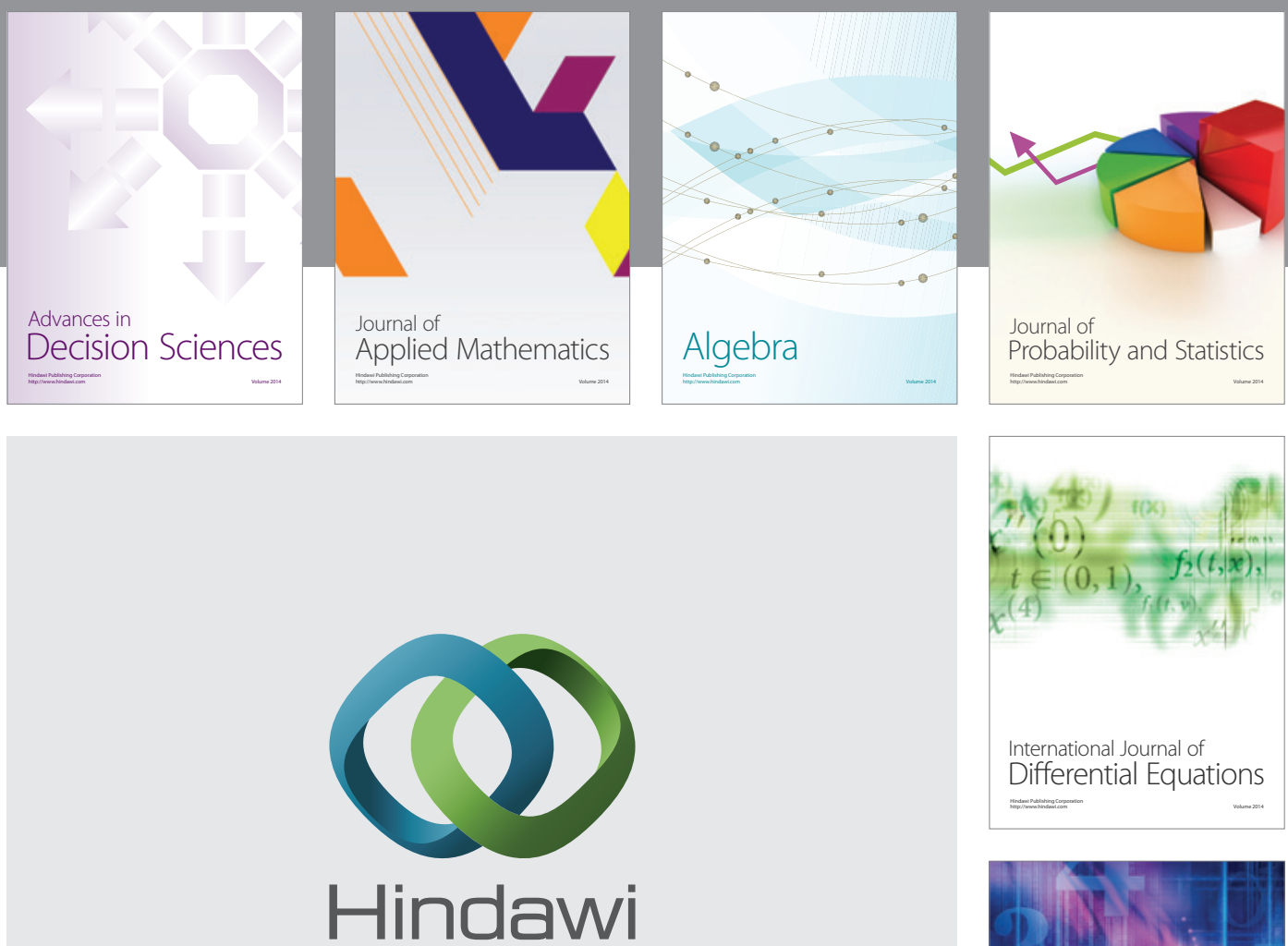

Submit your manuscripts at http://www.hindawi.com
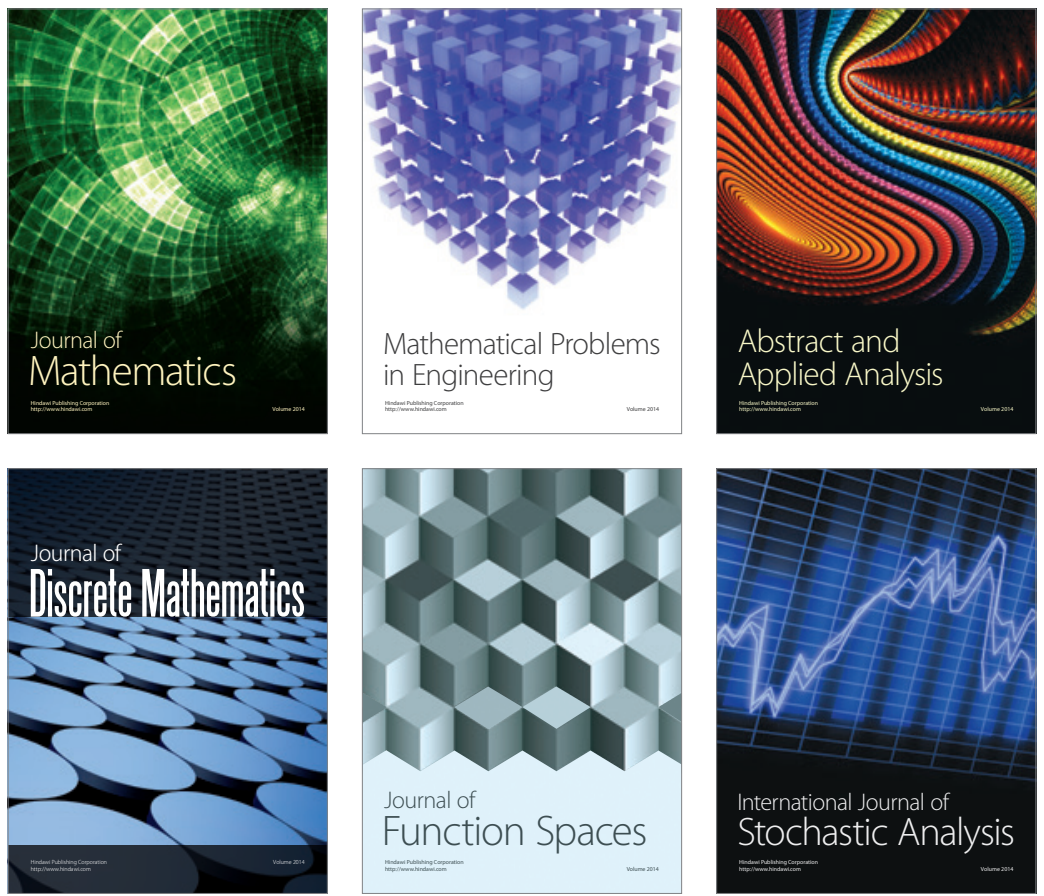

Journal of

Function Spaces

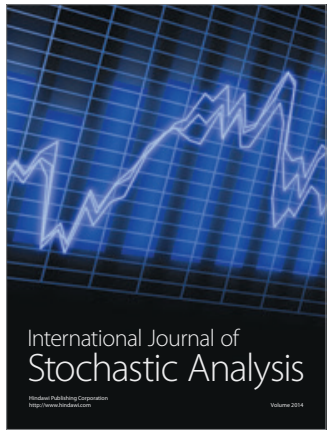

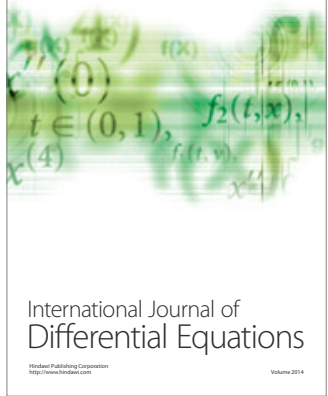
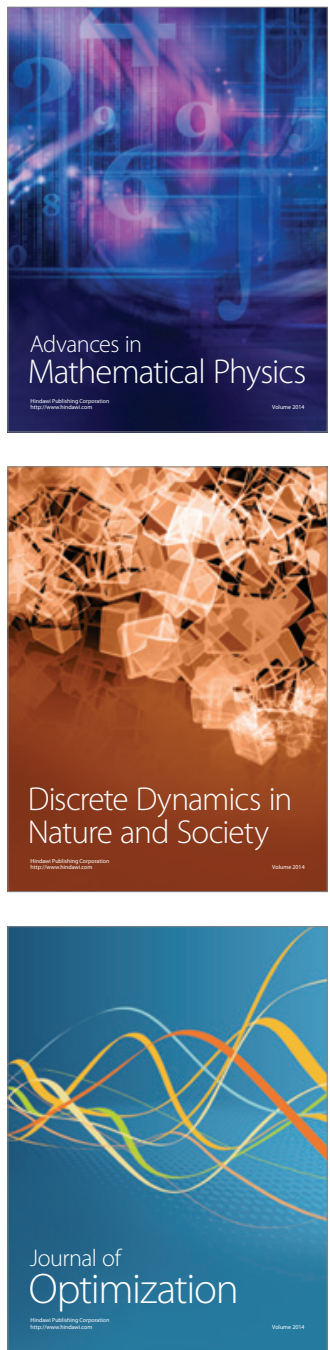